hep-ph/9805297

TTP 98-16

HD-THEP 98-18

August 10, 2018

\title{
Evolution of the Light-Cone Distribution Function for a Heavy Quark
}

\author{
Christopher Balzereit and Thomas Mannel \\ Institut für Theoretische Teilchenphysik, Universität Karlsruhe, \\ D - 76128 Karlsruhe, Germany \\ Wolfgang Kilian \\ Institut für Theoretische Physik, Universität Heidelberg, \\ D - 69120 Heidelberg, Germany
}

\begin{abstract}
We compute the one-loop anomalous dimension for the light cone distribution function of a heavy quark and solve the corresponding evolution equation analytically. Some implications of the results for inclusive $B$ decays are discussed.
\end{abstract}




\section{Introduction}

The heavy mass expansion for inclusive decays of heavy hadrons has become a generally accepted tool. Although there are hints that there could be problems in the calculation of lifetimes (the $\Lambda_{b}$-lifetime) within this framework, the $1 / m_{Q}$ expansion seems to be an appropriate method to access semileptonic processes [1, 2, 3].

However, if one is aiming at a complete description of the spectra (i.e. energy of the charged lepton $E_{\ell}$ or the hadronic invariant mass $\sqrt{Q_{H}^{2}}$ ) in general problems are encountered in the endpoint region, where $Q_{H}^{2}$ becomes small. Typically this invariant mass is of the order $m_{B}$, the mass of the decaying hadron. This is true in almost all phase space; however, getting closer to the endpoint it has become popular to distinguish two regions. The first region is the one with very small $Q_{H}^{2} \sim \Lambda_{Q C D}^{2}$ and also very small hadronic energy $v \cdot Q_{H} \sim \Lambda_{Q C D}$ (here $v$ is the velocity of the decaying hadron); here the proper description is a summation over the very few exclusive channels contributing in this region.

However, in the second region where the hadronic energy is still large $v \cdot Q_{H} \sim m_{B}$ but the invariant mass $Q_{H}^{2}$ becomes small, namely $Q_{H}^{2} \sim$ $\Lambda_{Q C D} m_{B}$, there is still the possibility to obtain a proper QCD description of the decay. In this case it has been shown [4, 5, 6] that one may resum the leading twist terms into a universal light-cone distribution function (or shape function) for the heavy quark. This function is entirely non-perturbative and may be written formally as a forward matrix element of a non-local operator. This nonlocal operator corresponds to the Fourier transform of the Wilson line [7, 9].

On the other hand, there are computable perturbative corrections which exhibit logarithmic singularities close to the endpoint region. To first order in $\alpha_{s}$ the leading contribution to the spectrum close to the endpoint is of the form $\alpha_{s} \ln \left(Q^{2}\right) / Q^{2}$, where $Q^{2}$ is now the partonic analogue of the hadronic invariant mass of the final state. Upon integration this yields the usual Sudakov logarithms.

Several methods have been proposed to combine both perturbative and non-perturbative contributions. At least for the doubly logarithmic terms this should be possible since they are process independent and hence could be assigned to the universal shape function. In addition, close to the endpoint, a resummation of the double logarithms becomes necessary, since these terms become large. Such a resummation may be achieved by setting up an 
evolution equation for the shape function as it has been proposed in [8, 9].

In the present note we use such an evolution equation and solve it analytically by putting in the one loop result for the anomalous dimensions. In the next section we express the hadronic invariant mass spectra in terms of the shape function, thereby defining a limit in which the leading twist dominates. In section 3 we consider the renormalization of the shape function and set up an evolution equation and derive the anomalous dimension in section 4. Finally we solve the evolution equation analytically and discuss the implications for the evolution of the moments of the shape function.

\section{Large energy limit of hadronic invariant mass spectra}

In inclusive decays such as $B \rightarrow X_{s}+\gamma$ and $B \rightarrow X_{u}+l+\nu_{l}$ the effective hamiltonian takes the general form

$$
\mathcal{H}_{\text {eff }}=j_{\mu} H^{\mu},
$$

where $H^{\mu}$ is a hadronic current and $j^{\mu}$ is either leptonic or photonic.

We shall in the following consider the invariant mass spectrum of the final state hadrons. We denote with $Q_{H}^{2}$ the square of this invariant mass. The spectrum may be written as

$$
\frac{d \Gamma}{d Q_{H}^{2}}=\frac{1}{2 m_{B}} \int d \Phi_{\ell} \sum_{X} \int d \Phi_{X}(2 \pi)^{4} \delta^{4}\left(P-q-P_{X}\right) \delta\left(Q_{H}^{2}-P_{X}^{2}\right) L_{\mu \nu} H^{\mu \nu},
$$

where phase space integration over the leptonic and hadronic total momentum $q, P_{X}$ is denoted by $d \Phi_{\ell, X}$, respectively.

The leptonic (or photonic) and hadronic tensors are defined as

$$
\begin{aligned}
L_{\mu \nu} & =\left\langle 0\left|j_{\mu}\right| l\right\rangle\left\langle l\left|j_{\nu}^{\dagger}\right| 0\right\rangle \\
H_{\mu \nu} & =\left\langle B(P)\left|H_{\mu}\right| X\right\rangle\left\langle X\left|H_{\nu}^{\dagger}\right| B(P)\right\rangle .
\end{aligned}
$$

The non-hadronic part can be calculated perturbatively and is treated in the

following as a known function of $q$. The object of our interest is the hadronic part,

$$
Q_{\mu \nu}=\sum_{X} \int d \Phi_{X}(2 \pi)^{4} \delta^{4}\left(P-q-P_{X}\right) H_{\mu \nu}
$$


which in general cannot be determined perturbatively. Proceeding along standard lines we have

$$
Q_{\mu \nu}=\int d^{4} x e^{i q x}\left\langle B(P)\left|H_{\mu}^{\dagger}(0) H_{\nu}(x)\right| B(P)\right\rangle
$$

The hadronic current is usually a bilinear function of two quark operators one of which is a heavy $b$ quark. Using the fact that the heavy mass $m_{b}$ sets a large scale compared to $\Lambda_{Q C D}$ we may set up an operator product expansion in the usual way. We write

$$
H_{\mu}=\bar{q}(x) \Gamma_{\mu} b(x)=e^{-i m_{b} v x} \bar{q}(x) \Gamma_{\mu} b_{v}(x),
$$

where $q(x)$ represents a massless quark and the large part of the $b$-quark momentum has been explicitely removed from the field by a phase redefinition. This leaves us with

$$
Q_{\mu \nu}=\int d^{4} x e^{-i x\left(m_{b} v-q\right)}\left\langle B(P)\left|\bar{b}_{v}(0) \Gamma_{\mu}^{\dagger} q(0) \bar{q}(x) \Gamma_{\nu} b_{v}(x)\right| B(P)\right\rangle,
$$

In the following we are interested in the endpoint region, which is defined by a specific kinematical limit denoted large-energy limit. We shall define this limit first in terms of the partonic total momentum of the final state

$$
Q^{\mu}=m_{b} v^{\mu}-q^{\mu}
$$

corresponding to the partonic invariant mass $Q^{2}$ and the partonic energy $v \cdot Q$. In the endpoint region the total energy $v \cdot Q$ of the final state scales with the heavy quark mass $m_{b}$ while the invariant mass $Q^{2}$ is of $\mathcal{O}\left(\Lambda_{Q C D} m_{b}\right)$ such that the light-cone component $k_{+}$of the total final state momentum remains finite of $\mathcal{O}\left(\Lambda_{Q C D}\right)$ :

$$
\begin{aligned}
m_{b} & \rightarrow \infty \\
v \cdot Q & \rightarrow \infty \\
\frac{2 v \cdot Q}{m_{b}} & =\text { const. } \\
k_{+} & =-\frac{Q^{2}}{2 v \cdot Q}=\text { const. } \\
\frac{Q^{2}}{m_{b}^{2}} & \rightarrow 0
\end{aligned}
$$


We shall relate the partonic variables to the hadronic ones at the end of the section.

It has been shown [5] that in the limit (10) the leading contribution may be obtained by contracting the light quark

$$
\begin{aligned}
Q_{\mu \nu}= & \int d^{4} x \int \frac{d^{4} k}{(2 \pi)^{4}} \Theta\left(k_{0}\right) \delta\left(k^{2}\right) e^{-i x(Q-k)} \\
& \left\langle B(P)\left|\bar{b}_{v}(0) \Gamma_{\mu}^{\dagger} \not k \Gamma_{\nu} \mathcal{P} \exp \left[-i \int_{0}^{x} d x \cdot A(x)\right] b_{v}(x)\right| B(P)\right\rangle .
\end{aligned}
$$

This expression may be Taylor-expanded around $x=0$ and the expansion resummed to yield formally

$$
\begin{aligned}
Q_{\mu \nu}= & \int \frac{d^{4} k}{(2 \pi)^{4}} \Theta\left(k_{0}\right) \delta\left(k^{2}\right) \\
& \left\langle B(P)\left|\bar{b}_{v}(0) \Gamma_{\mu}^{\dagger} \not k \Gamma_{\nu}(2 \pi)^{4} \delta^{4}(Q-k+i D) b_{v}(0)\right| B(P)\right\rangle .
\end{aligned}
$$

In the following, $\delta$-functions as in the preceding expression are defined by their Fourier transform, which involves a path-ordered exponential as in ([1]).

Performing the $k$-integration we get

$$
Q_{\mu \nu}=\left\langle B(P)\left|\bar{b}_{v}(0) \Gamma_{\mu}^{\dagger}(\not Q+i \not D) \Gamma_{\nu} \delta\left((Q+i D)^{2}\right) b_{v}(0)\right| B(P)\right\rangle .
$$

In the endpoint region (10) $Q$ is almost light-like and can be decomposed as

$$
Q=(v \cdot Q) n_{+}-k \quad \text { with } \quad v \cdot n_{+}=1, \quad n_{+}^{2}=0, \quad k=\mathcal{O}\left(\Lambda_{Q C D}\right) .
$$

Thus in (13) we can approximate $\left(i D_{+} \equiv n_{+} \cdot i D\right)$

$$
\begin{aligned}
(Q+i D)^{2} & \approx Q^{2}+2 v \cdot Q i D_{+} \\
\not+i \not D & \approx \varnothing \\
b_{v} & \approx h_{v}
\end{aligned}
$$

neglecting terms of $\mathcal{O}\left(\Lambda_{Q C D} / m_{b}\right)$ :

$$
\hat{Q}_{\mu \nu}=\left\langle B(v)\left|\bar{h}_{v}(0) \Gamma_{\mu}^{\dagger} \phi \Gamma_{\nu} \delta\left(Q^{2}+2 v \cdot Q i D_{+}\right) h_{v}(0)\right| B(v)\right\rangle
$$

For the perturbative calculation in the next section it is useful to rewrite $\hat{Q}_{\mu \nu}$ in terms of the imaginary part

$$
\hat{Q}_{\mu \nu}=\operatorname{Im} \frac{1}{\pi} \hat{T}_{\mu \nu},
$$


where

$$
\hat{T}_{\mu \nu}=-\left\langle B(v)\left|\bar{h}_{v}(0) \Gamma_{\mu}^{\dagger} \phi \Gamma_{\nu} \frac{1}{Q^{2}+2 v \cdot Q i D_{+}+i \epsilon} h_{v}(0)\right| B(v)\right\rangle .
$$

Using heavy-quark symmetry to disentangle the Dirac structure of this expression we finally arrive at

$$
\hat{Q}_{\mu \nu}=\frac{1}{2} \operatorname{Tr}\left\{\Gamma_{\mu}^{\dagger} \phi \Gamma_{\nu} P_{v}^{+}\right\} \int d k_{+} \delta\left(Q^{2}+2 v \cdot Q k_{+}\right) f\left(k_{+}\right),
$$

where $P_{v} \equiv(1+\not) / 2$.

Here we have introduced the shape function or light-cone distribution function for the heavy quark

$$
f\left(k_{+}\right)=\left\langle B(v)\left|\bar{h}_{v} \delta\left(k_{+}-i D_{+}\right) h_{v}\right| B(v)\right\rangle .
$$

The light-cone structure function $f\left(k_{+}\right)$measures the probability of finding a heavy quark with light-cone component $k_{+}$inside the B-meson and is a universal function.

Up to now we have worked in terms of partonic variables, while experimentally only hadronic variables are of interest. Introducing the hadronic light-cone variable in terms of the hadronic invariant mass squared $Q_{H}^{2}$ and the hadronic energy $v \cdot Q_{H}$ we have

$$
K_{+}=-\frac{Q_{H}^{2}}{2 v \cdot Q_{H}}=-\frac{Q^{2}+2 \bar{\Lambda}(v \cdot Q)}{2(v \cdot Q)+2 \bar{\Lambda}}=k_{+}-\bar{\Lambda}+\mathcal{O}\left(\bar{\Lambda}^{2} / m_{b}\right)
$$

we find that the support of $f\left(k_{+}\right)$is the interval $-\infty<k_{+}<\bar{\Lambda}$ such that $K_{+} \leq 0$. In reality, the spectrum is peaked near $k_{+}=0$. The shape function may directly be measured in a inclusive semileptonic or radiative decay by measuring the spectrum of the hadronic light-cone variable $K_{+}$.

\section{Renormalization of the light-cone structure function}

In the last section we have shown, that in the large-energy limit the integrated hadronic tensor $Q_{\mu \nu}$ can be approximated by the quantity $\hat{Q}_{\mu \nu}$ defined in the large energy limit of QCD. Up to now only non-perturbative corrections resummed in the structure function are included. 
To really establish the approximation we have to check that this also works perturbatively, since it is well known that in the kinematical region of small invariant mass $Q^{2}$ the perturbative spectrum is plagued by large logarithmic corrections. Therefore we should require that order by order in perturbation theory the leading IR singularities of $Q_{\mu \nu}$ arising in the largeenergy limit should be reproduced by $\hat{Q}_{\mu \nu}$. The leading logarithms are universal and hence we may assign them to be perturbative corrections to the structure function.

In order to identify the relevant IR singularities which have to be reproduced by perturbative corrections to the structure function we shall first consider the hadronic invariant mass spectrum calculated in full QCD in the large-energy limit. The relevant terms of the one loop contribution can be generically written as

$$
\frac{d \Gamma^{(\text {pert })(1)}}{d Q^{2}} \approx\left(\frac{\alpha_{s}}{\pi}\right)\left[\left(\frac{\ln \left(Q^{2} / m_{b}^{2}\right)}{k_{+}}\right)_{+} f_{1}\left(Q^{2} / m_{b}^{2}\right)+\left(\frac{1}{k_{+}}\right)_{+} f_{2}\left(Q^{2} / m_{b}^{2}\right)\right]
$$

where the real functions $f_{i}(\rho)$ are regular in the limit $\rho \rightarrow 0$ with $k_{+}$fixed. From this we easily identify the IR singularity as the $\ln \left(Q^{2} / m_{b}^{2}\right)$-term since $k_{+}$is held fixed in the large-energy limit. If the large-energy limit makes sense in perturbation theory, we have to require that exactly this term is reproduced by the $\mathcal{O}\left(\alpha_{s}\right)$-corrections to the light-cone distribution function.

The origin of this logarithmic divergence in the spectrum can be traced back to double logarithms of the correlator $T^{\mu \nu}$. Thus to one-loop order the double logarithmic terms in $T^{\mu \nu}$ and $\hat{T}^{\mu \nu}$ have to match in the large-energy limit. It therefore suffices to look for the most singular Feynman integrals contributing to the correlators at $\mathcal{O}\left(\alpha_{s}\right)$.

The one-loop corrections to the shape function consist of vertex diagrams, self-energy contributions and a box-type diagram. However, in full QCD the UV divergencies of the self-energy diagrams cancel with the UV divergency of the vertex diagram because of current conservation. For the shape function divergent pieces appear in the self energy of the heavy quark which will be taken into account below. The self-energy insertion into the light-cone propagator does not contribute. Thus, we can restrict ourselves to the vertex diagrams shown in figure 1 . 


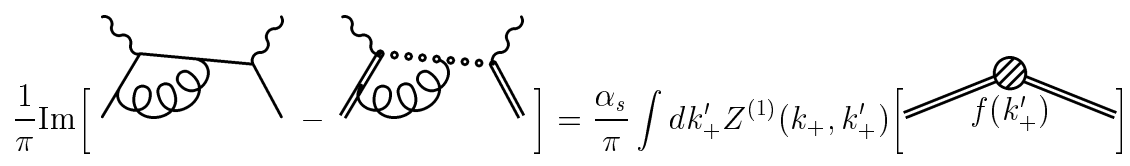

Figure 1: Renormalization of the structure function. The plain line denotes a massive or massless QCD propagator, the double plain line a heavy quark propagator, the dotted line a light-cone propagator. The shaded blob on the r.h.s. symbolizes the insertion of the structure function as a nonlocal operator.

The part of the vertex correction in full QCD (first term in Fig.11) responsible for double logarithms reads

$$
\begin{aligned}
V_{(\text {sing })=}^{\mu \nu}= & i g_{s}^{2} C_{F} 2 \frac{\Gamma^{\dagger \mu} \not \Gamma^{\nu}}{k_{+}^{\prime}-k_{+}+i \epsilon} \\
& \int \frac{d^{D} \ell}{(2 \pi)^{D}} \frac{1}{\left(v \cdot \ell+v \cdot k^{\prime}+\frac{1}{2 m}\left(\ell+k^{\prime}\right)^{2}\right) \ell^{2}\left[\left(\ell+k^{\prime}+Q\right)^{2}+i \epsilon\right]},
\end{aligned}
$$

where $k^{\prime}$ is the residual momentum of the heavy quark and the integration measure indicates dimensional regularization with $D=4-\epsilon$.

On the other hand, we can directly compute the corrections to the shape function either by reading off the propagator from (17) or by the usual way of calculating corrections to Wilson lines [7]. The corresponding expression is

$$
\begin{aligned}
\hat{V}_{(\text {sing })=}^{\mu \nu}= & i g_{s}^{2} C_{F} 2 \frac{\Gamma^{\dagger \mu} \not \Gamma^{\nu}}{k_{+}^{\prime}-k_{+}+i \epsilon} \\
& \int \frac{d^{D} \ell}{(2 \pi)^{D}} \frac{1}{\left(v \cdot \ell+v \cdot k^{\prime}\right) \ell^{2}\left(Q^{2}+2 v \cdot Q\left(\ell_{+}+k_{+}^{\prime}\right)+i \epsilon\right)}
\end{aligned}
$$

in which the leading logarithms have to match the full QCD result (22).

Evaluating the corresponding integrals $I$ and $\hat{I}$ to double logarithmic 
accuracy and supressing constant terms we get

$$
\begin{aligned}
I & =-\frac{i}{(4 \pi)^{2}} \frac{1}{v \cdot Q} \frac{1}{2} \ln ^{2}\left(\frac{k_{+}-k_{+}^{\prime}-i \epsilon}{\mu}\right) \\
\hat{I} & =-\frac{i}{(4 \pi)^{2}}(4 \pi)^{\frac{\epsilon}{2}} \frac{1}{v \cdot Q} \Gamma(1-\epsilon) \Gamma(\epsilon) \Gamma\left(\frac{\epsilon}{2}\right)\left(\frac{k_{+}-k_{+}^{\prime}-i \epsilon}{\mu}\right)^{-\epsilon} \\
& =-\frac{i}{(4 \pi)^{2}} \frac{1}{v \cdot Q}\left[\frac{2}{\epsilon^{2}}-\frac{2}{\epsilon} \ln \left(\frac{k_{+}-k_{+}^{\prime}-i \epsilon}{\mu}\right)+\frac{c}{\epsilon}+\ln ^{2}\left(\frac{k_{+}-k_{+}^{\prime}-i \epsilon}{\mu}\right)\right],
\end{aligned}
$$

where

$$
c=\ln (4 \pi)-\gamma_{E} .
$$

Note that (24) is valid up to single logarithmic corrections.

Due to the presence of both UV and collinear divergencies, a $1 / \epsilon^{2}$ pole appears. However, both singularities are of UV nature in the sense that the integral converges for $\epsilon>0$ and can therefore be removed by renormalization, as will be explained below.

In the difference of both integrals

$$
\begin{aligned}
\Delta I & =I-\hat{I} \\
& =-\frac{i}{(4 \pi)^{2}} \frac{1}{v \cdot Q}\left[-\frac{2}{\epsilon^{2}}+\frac{2}{\epsilon} \ln \left(\frac{k_{+}-k_{+}^{\prime}-i \epsilon}{\mu}\right)-\frac{c}{\epsilon}-\frac{1}{2} \ln ^{2}\left(\frac{k_{+}-k_{+}^{\prime}-i \epsilon}{\mu}\right)\right]
\end{aligned}
$$

the double logarithms do not cancel.

The operator to be renormalized [i.e., the operator defining the light-cone distribution function (19)] is of nonlocal nature. Hence, we can introduce an integration over $k_{+}^{\prime}$, which plays the role of a summation over a continuous operator basis labeled by $k_{+}^{\prime}$, to absorb the local as well as the nonlocal UV divergencies in (27) into a renormalized distribution function.

Furthermore, the difference of integrals (27) has a finite part. In order to reproduce the amplitudes (defined in a definite scheme, e.g. $M S$ ) of full QCD in the effective theory, these parts, the matching corrections, have to be included in the renormalization constants. In ordinary effective theories such as HQET, such finite matching corrections will not affect the scaling behavior to one-loop order, so they have to be taken into account explicitly only from two-loop order on. By contrast, in the present case the finite matching corrections

$$
\Delta I_{\text {finite }}=\frac{i}{(4 \pi)^{2}} \frac{1}{2 v \cdot Q} \ln ^{2}\left(\frac{k_{+}-k_{+}^{\prime}-i \epsilon}{\mu}\right)
$$


explicitly depend on the renormalization scale $\mu$ and therefore modify the scaling behavior already at one-loop order円.

In order to find the renormalization kernel, we take the imaginary part of the difference of vertex corrections to the correlators $T$ and $\hat{T}$ resulting from (27), keeping only UV-poles and double logarithms:

$$
\begin{aligned}
\Delta Q^{\mu \nu}= & \frac{1}{\pi} \operatorname{Im}\left(V_{(\text {sing })}^{\mu \nu}-\hat{V}_{(\text {sing })}^{\mu \nu}\right) \\
= & \frac{\alpha_{s}}{\pi} \frac{1}{2} \operatorname{Tr}\left\{\Gamma^{\mu \dagger} \phi \Gamma^{\nu} P_{v}^{+}\right\} \int d k_{+} \delta\left(Q^{2}+2 v \cdot Q k_{+}\right) \\
& \times \int d k_{+}^{\prime}\left[-Z^{(1)}\left(k_{+}, k_{+}^{\prime}, \mu\right)_{M S^{\prime}}-\frac{C_{F}}{\epsilon} \delta\left(k_{+}-k_{+}^{\prime}\right)\right] f^{(\text {bare })}\left(k_{+}^{\prime}\right)
\end{aligned}
$$

The second term in square brackets accounts for wave function renormalization of the heavy-quark fields. The one-loop contribution to vertex renormalization is given by $\mathrm{f}$

$$
\begin{aligned}
Z^{(1)}\left(k_{+}, k_{+}^{\prime}, \mu\right)_{M S^{\prime}}= & C_{F}\left(\frac{2}{\epsilon^{2}}+\frac{c-1}{\epsilon}\right) \delta\left(k_{+}-k_{+}^{\prime}\right) \\
& -C_{F} \frac{2}{\epsilon} \frac{d}{d k_{+}^{\prime}}\left[\Theta\left(k_{+}^{\prime}-k_{+}\right) \ln \left(\frac{\left|k_{+}^{\prime}-k_{+}\right|}{\mu}\right)\right] \\
& +\frac{1}{2} C_{F} \frac{d}{d k_{+}^{\prime}}\left[\Theta\left(k_{+}^{\prime}-k_{+}\right) \ln ^{2}\left(\frac{\left|k_{+}^{\prime}-k_{+}\right|}{\mu}\right)\right],
\end{aligned}
$$

which has to be understood in the distribution sense.

In this way we can define a renormalized shape function

$$
f\left(k_{+}, \mu\right)=\int d k_{+}^{\prime} Z\left(k_{+}, k_{+}^{\prime}, \mu\right)_{M S^{\prime}} f^{(\text {bare })}\left(k_{+}^{\prime}\right)
$$

where the one loop renormalization kernel reads

$$
Z\left(k_{+}, k_{+}^{\prime}, \mu\right)_{M S^{\prime}}=\delta\left(k_{+}-k_{+}^{\prime}\right)+\left(\frac{\alpha_{s}}{\pi}\right) Z^{(1)}\left(k_{+}, k_{+}^{\prime}, \mu\right)_{M S^{\prime}}
$$

\footnotetext{
${ }^{1}$ The appeareance of nonlocal divergencies and double logarithms in the matching corrections spoils the naive formulation of a large-energy effective theory (LEET) for exclusive processes, as has been observed in [11, 12].

${ }^{2}$ The renormalization prescription which matches QCD in the $M S$ scheme is denoted as $M S^{\prime}$.
} 
In what follows it will be convenient to switch to the $\overline{M S}^{\prime}$-scheme setting

$$
\frac{1}{\epsilon}=\frac{1}{\hat{\epsilon}}-\frac{1}{2} \ln (4 \pi)+\frac{1}{2} \gamma_{E}
$$

and keeping terms proportional to $1 / \hat{\epsilon}^{2}$ and $1 / \hat{\epsilon}$ in the renormalization-kernel:

$$
\begin{aligned}
Z^{(1)}\left(k_{+}, k_{+}^{\prime}, \mu\right) \overline{M_{S}} & =C_{F}\left(\frac{2}{\hat{\epsilon}^{2}}-\frac{c+1}{\hat{\epsilon}}\right) \delta\left(k_{+}-k_{+}^{\prime}\right) \\
& -C_{F} \frac{2}{\hat{\epsilon}} \frac{d}{d k_{+}^{\prime}}\left[\Theta\left(k_{+}^{\prime}-k_{+}\right) \ln \left(\frac{\left|k_{+}^{\prime}-k_{+}\right|}{\mu}\right)\right] \\
& +\frac{1}{2} C_{F} \frac{d}{d k_{+}^{\prime}}\left[\Theta\left(k_{+}^{\prime}-k_{+}\right) \ln ^{2}\left(\frac{\left|k_{+}^{\prime}-k_{+}\right|}{\mu}\right)\right]
\end{aligned}
$$

Then to one loop order

$$
\hat{Q}_{\mu \nu}=\frac{1}{2} \operatorname{Tr}\left\{\Gamma_{\mu}^{\dagger} \phi \Gamma_{\nu} P_{v}^{+}\right\} \int d k_{+} \delta\left(Q^{2}+2 v \cdot Q k_{+}\right) f\left(k_{+}, M\right)
$$

is UV-finite. Here $M$ is a scale of the order $v \cdot Q$ which is a perturbative scale. As a consequence of the finite renormalization in (35) also the correct collinear singularity is reproduced.

\section{Evolution equation}

As a consequence of the renormalization procedure the structure function now depends on both the renormalization scale $\mu$ and the renormalizationscheme $\left(\overline{M S}^{\prime}\right)$ and obeys the evolution equation

$$
\frac{d}{d \ln \mu} f\left(k_{+}, \mu\right)=\int d k_{+}^{\prime} \Gamma\left(k_{+}, k_{+}^{\prime}, \mu\right) f\left(k_{+}^{\prime}, \mu\right) .
$$

The evolution kernel $\Gamma\left(k_{+}, k_{+}^{\prime}, \mu\right)$ in the $\overline{M S}^{\prime}$-scheme ${ }^{\beta}$ is defined implicitly by

$$
\int d k_{+}^{\prime \prime} \Gamma\left(k_{+}, k_{+}^{\prime \prime}, \mu\right) Z\left(k_{+}^{\prime \prime}, k_{+}^{\prime}, \mu\right)_{\overline{M S}}=\frac{d}{d \ln \mu} Z\left(k_{+}, k_{+}^{\prime}, \mu\right)_{\overline{M S}},
$$

\footnotetext{
${ }^{3}$ In what follows this scheme dependence of the evolution kernel is understood.
} 
where

$$
\frac{d}{d \ln \mu} \equiv \frac{\partial}{\partial \ln \mu}-2 \alpha_{s}\left(\frac{\alpha_{s}}{\pi} \beta_{0}+\ldots\right) \frac{\partial}{\partial \alpha_{s}}
$$

with $\beta_{0}=\left(33-2 n_{f}\right) / 12$. Thus, to one-loop order the evolution kernel is given by the negative coefficient of the $1 / \epsilon$-pole term in $Z^{(1)}\left(k_{+}, k_{+}^{\prime}, \mu\right) \overline{M S}^{\prime}$ and the partial $\ln (\mu)$-derivative of its finite partf:

$$
\begin{aligned}
\Gamma\left(k_{+}, k_{+}^{\prime}, \mu\right) & =\left(\frac{\alpha_{s}(\mu)}{\pi}\right) \Gamma^{(1)}\left(k_{+}, k_{+}^{\prime}, \mu\right) \\
& =\left(\frac{\alpha_{s}}{\pi}\right) C_{F}\left(\delta\left(k_{+}^{\prime}-k_{+}\right)+\frac{d}{d k_{+}^{\prime}}\left[\Theta\left(k_{+}^{\prime}-k_{+}\right) \ln \left(\frac{\left|k_{+}^{\prime}-k_{+}\right|}{\mu}\right)\right]\right)
\end{aligned}
$$

In order to solve the evolution equation we Fourier transform both the structure function and the evolution kernel:

$$
\begin{aligned}
\tilde{f}(\xi, \mu) & =\int \frac{d k_{+}}{2 \pi} f\left(k_{+}, \mu\right) e^{i k_{+} \xi} \\
\tilde{\Gamma}^{(1)}(\xi, \mu) & =\int \frac{d k_{+}}{2 \pi} \Gamma^{(1)}\left(k_{+}, 0, \mu\right) e^{i k_{+} \xi} \\
& =\frac{C_{F}}{2 \pi}\left\{1-\ln (|\xi \mu|)-i \frac{\pi}{2}(\Theta(\xi)-\Theta(-\xi))\right\}
\end{aligned}
$$

Then the evolution equation (37) reads

$$
\frac{d}{d \ln \mu} \tilde{f}(\xi, \mu)=\left(\frac{\alpha_{s}}{\pi}\right)(2 \pi) \tilde{\Gamma}^{(1)}(\xi, \mu) \tilde{f}(\xi, \mu)
$$

and can be solved easily

$$
\tilde{f}(\xi, \mu)=\tilde{U}\left(\xi, \mu, \mu_{0}\right) \tilde{f}\left(\xi, \mu_{0}\right)
$$

where

$$
\tilde{U}\left(\xi, \mu, \mu_{0}\right)=K\left(\mu, \mu_{0}\right) \exp \left(i \frac{\pi}{2} \omega \varepsilon(\xi)\right)\left|\mu_{0} \xi\right|^{\omega}
$$

\footnotetext{
${ }^{4}$ Note that the double pole in $Z^{(1)}\left(k_{+}, k_{+}^{\prime}, \mu\right) \overline{M S^{\prime}}$ cancels with the explicit $\mu$-derivative of the single pole-term:

$$
\frac{d}{d \ln \mu} \frac{d}{d k_{+}^{\prime}}\left[\Theta\left(k_{+}^{\prime}-k_{+}\right) \ln \left(\frac{\left|k_{+}^{\prime}-k_{+}\right|}{\mu}\right)\right]=-\delta\left(k_{+}^{\prime}-k_{+}\right)
$$


with

$$
\omega=\frac{C_{F}}{2 \beta_{0}} \ln \left(\frac{\alpha_{s}(\mu)}{\alpha_{s}\left(\mu_{0}\right)}\right), \quad \varepsilon(\xi)=\Theta(\xi)-\Theta(-\xi)
$$

and

$$
K\left(\mu, \mu_{0}\right)=\left(\frac{\mu}{\mu_{0}}\right)^{-\frac{C_{F}}{2 \beta_{0}}}\left(\frac{\alpha_{s}(\mu)}{\alpha_{s}\left(\mu_{0}\right)}\right)^{-\frac{C_{F}}{2 \beta_{0}}\left[1+\frac{\pi}{2 \beta_{0} \alpha_{s}\left(\mu_{0}\right)}\right]} .
$$

Transforming back to momentum space we get

$$
f\left(k_{+}, \mu\right)=\int d k_{+}^{\prime} U\left(k_{+}, k_{+}^{\prime} ; \mu, \mu_{0}\right) f\left(k_{+}^{\prime}, \mu_{0}\right)
$$

where

$$
U\left(k_{+}, k_{+}^{\prime} ; \mu, \mu_{0}\right)=-K\left(\mu, \mu_{0}\right) \Gamma(1+\omega) \mu_{0}^{\omega} \frac{\sin \pi \omega}{\pi} \frac{\Theta\left(k_{+}^{\prime}-k_{+}\right)}{\left(k_{+}^{\prime}-k_{+}\right)^{1+\omega}} .
$$

Note that the tree-level result is recovered from (50) by letting $\mu=\mu_{0}$ and performing the limit $\omega \rightarrow-0$ carefully, since

$$
\lim _{\omega \rightarrow-0} \frac{\sin \pi \omega}{\pi} \frac{\Theta\left(k_{+}^{\prime}-k_{+}\right)}{\left(k_{+}^{\prime}-k_{+}\right)^{1+\omega}}=-\delta\left(k_{+}^{\prime}-k_{+}\right) .
$$

Once radiative corrections in the definition of the light-cone structure function are included, we are in the position to study the invariant mass spectrum. To this end we insert (49) into (36), choose for $\mu$ a high energy scale $M=\mathcal{O}(v \cdot Q)$ and let $\mu_{0}=\mu$

$$
\hat{Q}_{\mu \nu}=\frac{1}{2} \operatorname{Tr}\left\{\Gamma_{\mu}^{\dagger} \phi \Gamma_{\nu} P_{v}^{+}\right\} \int d k_{+}^{\prime} U\left(k_{+}, k_{+}^{\prime} ; M, \mu\right) f\left(k_{+}^{\prime}, \mu\right)
$$

The coefficient function $U\left(k_{+}, k_{+}^{\prime} ; M, \mu\right)$ is analogous to the set of Wilson coefficients $C_{i}(M, \mu)$ in local effective field theories.

Note that, by definition, the convolution of the renormalized structure function with the coefficient function is independent of the choice of the renormalization scale $\mu$. At the low energy scale $\mu=\mathcal{O}\left(\Lambda_{Q C D}\right)$ the structure function is only weakly affected by perturbative corrections and can be safely 
considered as a pure non-perturbative object which may be described by a model [13]:

$$
f\left(k_{+}, \Lambda_{Q C D}\right)=f^{(\text {model })}\left(k_{+}\right)
$$

Putting everything together, the renormalization group improved hadronic tensor becomes:

$$
\begin{aligned}
\hat{Q}_{\mu \nu}= & -\frac{1}{2(2 v \cdot Q)} K(M, \mu) \Gamma(1+\omega) \mu^{\omega} \frac{\sin \pi \omega}{\pi} \operatorname{Tr}\left\{\Gamma_{\mu}^{\dagger} \phi \Gamma_{\nu} P_{v}^{+}\right\} \\
& \int d k_{+}^{\prime} \frac{\Theta\left(k_{+}^{\prime}-k_{+}\right)}{\left(k_{+}^{\prime}-k_{+}\right)^{1+\omega}} f^{(\text {model })}\left(k_{+}^{\prime}\right)
\end{aligned}
$$

\section{Evolution of Moments}

It is well known that the moments of the light-cone structure function may be related to matrix elements of local operators. The moments satisfy the relation

$$
M_{n}=\int d k_{+}\left(k_{+}\right)^{n} f\left(k_{+}\right)=\left\langle B(v)\left|\bar{h}_{v}\left(i D_{+}\right)^{n} h_{v}\right| B(v)\right\rangle
$$

in particular we have $M_{0}=1$ and $M_{1}=0$ due to the normalization and the equations of motion.

However, since the light-cone distribution funtion evolves with a change of scale, also the moments depend on the scale. The moments at the scale $\mu$ are given by

$M_{n}(\mu)=\int d k_{+}\left(k_{+}\right)^{n} f\left(k_{+}, \mu\right)=\int d k_{+}\left(k_{+}\right)^{n} \int d k_{+}^{\prime} U\left(k_{+}, k_{+}^{\prime}, \mu, \mu_{0}\right) f\left(k_{+}^{\prime}, \mu_{0}\right)$

Since the kernel $U\left(k_{+}, k_{+}^{\prime}, \mu, \mu_{0}\right)$ depends only on the difference of $k_{+}$and $k_{+}^{\prime}$ one may reexpress these moments in terms of the moments at scale $\mu_{0}$ by a simple change of variables. One obtains

$$
M_{n}(\mu)=\sum_{j=0}^{n} \frac{n !}{j !(n-j) !} M_{j}\left(\mu_{0}\right) \int d k_{+}\left(k_{+}\right)^{n-j} U\left(k_{+}, 0, \mu, \mu_{0}\right)
$$

which means that under renormalization the $n^{\text {th }}$ moment will depend on all the moments $M_{j}$ with $j \leq n$ at the lower scale. 
However, due to the power type behaviour in the variable $k_{+}-k_{+}^{\prime}$ of the evolution kernel $U\left(k_{+}, k_{+}^{\prime}, \mu, \mu_{0}\right)$ the integral in (58) does not converge, since the region of integration is $-\infty \leq k_{+} \leq 0$ and hence it has to be regularized. If we regularize the integral by restricting the integration region to $-\Lambda \leq$ $k_{+} \leq 0$ with some cut off $\Lambda$, the dependence on $\Lambda$ can be already guessed from dimensional analysis. More precisely, the moments $M_{n}(\mu)$ exhibit power divergencies where the dependence on $\Lambda$ is given by

$$
M_{n}(\mu)=\sum_{j=0}^{n} M_{j}\left(\mu_{0}\right) C_{n, j}\left(\mu, \mu_{0}\right)\left(\frac{\mu_{0}}{\Lambda}\right)^{\omega} \Lambda^{n-j}
$$

where from (50) we get

$$
C_{n, j}\left(\mu, \mu_{0}\right)=-\frac{n !}{j !(n-j) !} K\left(\mu, \mu_{0}\right) \Gamma(1+\omega) \frac{\sin \pi \omega}{\pi} .
$$

To interpret this result it is instructive to consider the first few moments. The normalization receives a multiplicative renormalization

$$
M_{0}(\mu)=M_{0}\left(\mu_{0}\right) C_{0,0}\left(\mu, \mu_{0}\right)\left(\frac{\mu_{0}}{\Lambda}\right)^{\omega}
$$

while the first moment receives contributions from both $M_{0}\left(\mu_{0}\right)$ and $M_{1}\left(\mu_{0}\right)$ :

$$
M_{1}(\mu)=M_{1}\left(\mu_{0}\right) C_{1,1}\left(\mu, \mu_{0}\right)\left(\frac{\mu_{0}}{\Lambda}\right)^{\omega}+\Lambda M_{0}\left(\mu_{0}\right) C_{1,0}\left(\mu, \mu_{0}\right)\left(\frac{\mu_{0}}{\Lambda}\right)^{\omega}
$$

However, the first moment is

$$
M_{1}=\left\langle B(v)\left|\bar{h}_{v}(i v \cdot D) h_{v}\right| B(v)\right\rangle
$$

which according to the equations of motion should vanish. This is true, if the pole mass of the heavy quark is taken as the expansion parameter, such that no residual mass term appears. The choice of a different mass definition $m \rightarrow m+\delta m$ will modify the equation of motion into

$$
(i v \cdot D) h_{v}=\delta m h_{v}
$$

and hence the running of the first moment implies a change in the pole mass definition due to the power divergence

$$
\delta m=\Lambda C_{1}\left(\mu, \mu_{0}\right)\left(\frac{\mu_{0}}{\Lambda}\right)^{\omega} .
$$


In principle this relation can be used to fix the value of the cut-off in terms of the pole mass; once this is done, all higher moments can be computed in terms of the pole mass at the lower scale $\mu_{0}$.

Although the shape function is an object entirely defined in HQET and hence should be independent of the mass $m_{b}$, in this indirect way a mass dependence comes into the game, at least if we insist to interpret the moments in terms of the matrix elements (56).

\section{Conclusions}

In this paper we have proposed a method to combine perturbative and nonperturbative contributions to hadronic invariant mass spectra in the endpoint region where the invariant mass $Q_{H}^{2}$ becomes small of the order $\mathcal{O}\left(\Lambda_{Q C D} m_{B}\right)$. At tree level the non-perturbative corrections of leading twist are resummed into a universal light-cone distribution function. However, taking radiative corrections into account, this function has to be renormalized. In order to match the leading IR-singularity of the QCD spectrum, we have chosen a renormalization scheme which except standard UV-renormalization provides corrections of the IR-behaviour of the structure function adding proper finite terms to the renormalization kernel. As a consequence of renormalization the renormalization scale dependence of the structure function is described by an evolution equation. We have computed the evolution kernel to one loop order, which deviates from the usual $\overline{M S}$-kernel, since we have included a finite renormalization in order to reproduce the infrared behaviour of full QCD.

The analytical solution of the evolution equation yields a resummation of logarithmic corrections and relates structure functions at different renormalization scales. The last property has then be used to include radiative corrections in the invariant mass spectrum in a manner common from usual renormalization group techniques.

As the structure function itself is scale dependent so are its moments. However, including radiative corrections the moments exhibit an UV-divergence which has been regularized by an hard cut off thereby mixing moments of different order. We fixed the cut off by relating the first moment to the definition of the pole mass. Once the value of the cut off is known, the scale dependence of all other moments is computable. 


\section{Acknowledgements}

We are grateful to M. Neubert and V. Smirnov for valuable discussions. This work was supported by the "Graduiertenkolleg: Elementarteilchenphysik and Beschleunigern" and the "Forschergruppe: Quantenfeldtheorie, Computeralgebra und Monte Carlo Simulationen" of the Deutsche Forschungsgemeinschaft. W.K. is supported by German Bundesministerium für Bildung und Forschung (BMBF), Contract No. 05 6HD 91 P(0).

\section{References}

[1] A.F. Falk, M. Luke and M.J. Savage, Phys. Rev. D53, 2491 (1996).

[2] A.F. Falk, M. Luke and M.J. Savage, Phys. Rev. D53, 6316 (1996).

[3] A.F. Falk and M. Luke, Phys. Rev. D57, 424 (1998)

[4] M. Neubert, Phys. Rev. D49, 3392 and 4623 (1994).

[5] T. Mannel and M. Neubert, Phys. Rev. D50, 2037 (1994).

[6] I.I. Bigi, M.A. Shifman, N.G. Uraltsev and A.I. Vainshtein, Phys. Lett. B328, 431 (1994)

[7] G.P. Korchemsky and G. Sterman, Phys. Lett. B340, 96 (1994).

[8] A.G. Grozin and M. Neubert, Phys. Rev. D55, 272 (1997).

[9] A.G. Grozin and G.P. Korchemsky, Phys. Rev. D53, 1378 (1996).

[10] M.J. Dugan and B. Grinstein, Phys.Lett. B255, 583 (1991).

[11] U. Aglietti and G. Corbo, hep-ph 9803485.

[12] C. Balzereit, T. Mannel and V. Smirnov, in preparation.

[13] The BABAR Physics Book, SLAC-R-504, in preparation. 